\title{
Perfil clínico-epidemiológico de pacientes portadores de hanseníase em um município do Maranhão
}

\author{
Clinical-epidemiological profile of leprosy patients in a municipality of Maranhão
}

\section{Perfil clínico-epidemiológico de pacientes con leprosia en un municipio de Maranhão}

Patrícia Samara Ribeiro da Silva ${ }^{1 *}$, Naylanny Gonçalves Torres Cunha ${ }^{1}$, Larissa Silva Oliveira ${ }^{1}$, Maria Cleilda Araujo Santos ${ }^{1}$.

\section{RESUMO}

Objetivo: Analisar o perfil clínico-epidemiológico de pacientes portadores de hanseníase em um município maranhense no período de 2015 a 2017. Métodos: Trata-se de uma pesquisa epidemiológica, retrospectiva, de caráter descritivo e abordagem quantitativa, na qual foram utilizados dados secundários provenientes do Sistema de Informação de Agravos de Notificação (SINAN). Foi utilizado o software Microsoft Excel 2013 para a tabulação dos dado e elaboração de gráficos e tabelas. Resultados: No período de 2015 a 2017 foram notificados 265 casos de hanseníase, com predomínio no sexo masculino $(63 \%)$, na faixa etária de 30 a 39 anos $(20 \%)$, maior ocorrência de casos multibacilares $(89 \%)$, sendo a forma clínica dimorfa a mais frequente $(66,4 \%)$. Quanto ao modo de entrada, a maioria dos casos foram registrados como caso novo $(68,7 \%)$, o modo de detecção predominante foi a demanda espontânea (36\%) e no que se refere ao grau de incapacidade física no diagnóstico, a maior parte dos pacientes apresentou grau 0 de incapacidade (44\%). No tipo de saída, predominou a saída por cura $(54 \%)$. Conclusão: A hanseníase continua sendo um problema de saúde pública na região, uma vez que o município ainda é considerado hiperendêmico para a doença, conforme parâmetros estabelecidos pelo Ministério da Saúde.

Palavras-chave: Hanseníase, Perfil de saúde, Epidemiologia.

\begin{abstract}
Objective: To analyze the clinical-epidemiological profile of patients with leprosy in a municipality in Maranhão from 2015 to 2017. Methods: This is an epidemiological, retrospective, descriptive study with a quantitative approach, in which secondary data from of the Notifiable Diseases Information System (SINAN). Microsoft Excel 2013 software was used to tabulate data and create graphs and tables. Results: In the period from 2015 to 2017, 265 cases of leprosy were reported, with a predominance in males (63\%), aged between 30 and 39 years $(20 \%)$, the highest occurrence of multibacillary cases $(89 \%)$, being the dimorphic clinical form the most frequent $(66,4 \%)$. As for the entry mode, most cases were registered as a new case $(68,7 \%)$, the predominant mode of detection was spontaneous demand $(36 \%)$ and with regard to the degree of physical disability in the diagnosis, the highest part of the patients presented degree 0 of disability (44\%). In the type of exit, exit by cure predominated (54\%). Conclusion: Leprosy remains a public health problem in the region, since the municipality is still considered hyper-endemic for the disease, according to parameters established by the Ministry of Health.
\end{abstract}

Keywords: Leprosy, Health profile, Epidemiology.

\footnotetext{
1 Universidade Estadual do Maranhão (UEMA), Bacabal - MA.

*E-mail: patriciasamaras3@hotmail.com
} 


\section{RESUMEN}

Objetivo: Analizar el perfil clínico-epidemiológico de pacientes con lepra en un municipio de Maranhão de 2015 a 2017. Métodos: Estudio epidemiológico, retrospectivo, descriptivo con enfoque cuantitativo, en el que se obtienen datos secundarios de del Sistema de Información de Enfermedades de Notificación (SINAN). El software Microsoft Excel 2013 se usó para tabular datos y crear gráficos y tablas. Resultados: en el período de 2015 a 2017, se informaron 265 casos de lepra, con predominio en hombres (63\%), con edades comprendidas entre 30 y 39 años (20\%), siendo la mayor incidencia de casos multibacilares (89\%), siendo el forma clínica dimórfica la más frecuente $(66,4 \%)$. En cuanto al modo de entrada, la mayoría de los casos se registraron como un caso nuevo $(68,7 \%)$, el modo predominante de detección fue la demanda espontánea (36\%) y con respecto al grado de discapacidad física en el diagnóstico, el más alto parte de los pacientes presentaron grado 0 de discapacidad (44\%). En el tipo de salida, predomina la salida por cura (54\%). Conclusión: La lepra sigue siendo un problema de salud pública en la región, ya que el municipio todavía se considera hiperendémico para la enfermedad, según los parámetros establecidos por el Ministerio de Salud.

Palabras clave: Enfermedad de hansen, Perfil de salud, Epidemiología.

\section{INTRODUÇÃO}

A hanseníase é considerada uma das doenças mais antigas já descritas, com pistas de sua existência remontando ao período da antiguidade, sendo conhecida há mais de 3 ou 4 mil anos na Índia, na China e no Japão, havendo ainda relatos de sua presença no Egito desde 4300 anos a. C em um papiro da época do faraó Ramsés II (MACIEL LR e FERREIRA IN, 2014). É considerada um grave problema de saúde pública, sendo definida pelo Ministério da Saúde (2017b) como uma doença crônica e infectocontagiosa causada por uma bactéria chamada Mycobacterium leprae, que infecta a pele e os nervos periféricos, particularmente as células de Schwann, atingindo principalmente os nervos superficiais e troncos nervosos periféricos, podendo também afetar outros órgãos como olhos, mucosas, testículos, ossos, baço e fígado.

O diagnóstico de hanseníase é essencialmente clínico e epidemiológico e deve ser baseado na análise da história e condições de vida do paciente, bem como na realização do exame dermatoneurológico para identificação de lesões ou áreas de pele com alteração de sensibilidade e/ou comprometimento de troncos nervosos periféricos com alterações sensitivas e/ou motoras e/ou autonômicas.

Exames complementares como a baciloscopia e o exame histopatológico podem ser utilizados para auxiliar no diagnóstico da doença, porém, é importante destacar que os mesmos não definem diagnóstico e que deve-se sempre atentar para as manifestações clínicas apresentadas pelo paciente (BRASIL, 2016).

No âmbito da hanseníase, o profissional enfermeiro desempenha uma série de cuidados para garantir uma atenção integral e humanizada voltada ao paciente portador da doença, por meio da sistematização do cuidado de enfermagem, de modo que atua na prevenção da hanseníase, na identificação e avaliação de casos suspeitos, realiza a consulta de enfermagem, notifica os casos confirmados, avalia e registra o grau de incapacidade física em prontuários e formulários, orienta o paciente e a família para a realização do autocuidado, realiza o exame dermatoneurológico, faz a vacinação da BCG em todos os contatos intradomiciliares, além de gerenciar as atividades de controle da doença (COÊLHO LS, et al., 2015; NASCIMENTO GRC, et al., 2011).

Segundo a Organização Mundial da Saúde (OMS), no ano de 2016 foram detectados 214.783 casos novos de hanseníase em 143 países, apontando uma taxa de deteç̧ão de 2,9 casos por 100 mil habitantes. Nesse mesmo ano, foram notificados no Brasil 25.218 casos novos de hanseníase, com uma taxa de detecção de 12,2 casos para 100 mil habitantes. Tais dados demonstram que o país é considerado como de alta carga para a doença e o segundo com maior número de casos novos registrados no mundo (BRASIL, 2018). 
As taxas de detecção da hanseníase no Brasil apresentam tendência de redução, mas apesar disso esse comportamento não foi observado em todas as regiões brasileiras, dado que as regiões Norte, Nordeste e Centro- Oeste ainda possuem um alto padrão de endemicidade, apresentando, respectivamente, taxas de 29,65/100.000 habitantes, 22,72/100.000 habitantes e 44,30/100.000 habitantes, enquanto as regiões Sudeste e Sul detêm parâmetros médios de endemicidade, com taxas de 4,71/100.000 habitantes e 3,49/100.000 habitantes, respectivamente (BRASIL, 2017a).

No ano de 2015, a taxa de prevalência da hanseníase no Maranhão era de 3,76 casos por 10 mil habitantes, resultado que o colocou em terceiro lugar no ranking de casos entre as unidades da federação, o que possivelmente está relacionado à desigualdade social do estado, problema que acaba interferindo nas condições de saúde e acesso à informação por parte da população (BRASIL, 2016 apud MENDONÇA CAS, 2018). Considerando o contexto da hanseníase como problema de saúde pública e seu alto padrão de endemicidade, foi formulada a seguinte questão norteadora da pesquisa: Qual o perfil clínico-epidemiológico de pacientes portadores de hanseníase no município de Bacabal - MA no período de 2015 a 2017 ?

A realização do presente estudo permite que se tome conhecimento da situação da hanseníase no município, de modo a contribuir para a sua monitorização e para a elaboração e implementação de medidas que visem à redução do agravo e possibilitem o enfrentamento da doença.

Além disso, os resultados da pesquisa servirão de base para que os profissionais de saúde possam traçar estratégias que garantam um melhor acompanhamento dos portadores de hanseníase, dado que tais informações serão capazes de fornecer um direcionamento para a execução das ações de saúde a serem desenvolvidas com esses usuários. Nessa perspectiva, a pesquisa teve como objetivo analisar o perfil clínico-epidemiológico de pacientes portadores de hanseníase no município de Bacabal - MA no período de 2015 a 2017.

\section{MÉTODOS}

Trata-se de uma pesquisa epidemiológica, retrospectiva, de caráter descritivo e abordagem quantitativa, na qual foram utilizados dados secundários provenientes do Sistema de Informação de Agravos de Notificação (SINAN). A população-alvo do estudo foi composta por todos os pacientes portadores de hanseníase notificados no SINAN no período de 2015 a 2017 no município maranhense.

A coleta de dados foi realizada no mês de Julho de 2019 e os dados foram obtidos eletronicamente através do SINAN via Departamento de Informática do Sistema Único de Saúde (DATASUS), contemplando as seguintes variáveis para a pesquisa: número de casos de hanseníase notificados entre 2015 e 2017; sexo; faixa etária; classificação operacional; forma clínica; modo de entrada; modo de detecção; grau de incapacidade física no diagnóstico; e tipo de saída. Foram incluídos na pesquisa todos os dados referentes a casos de hanseníase em ambos os sexos e de todas as faixas etárias, notificados no SINAN no período de 2015 a 2017 no município, sendo excluídos os dados que estavam fora do período selecionado para o desenvolvimento do estudo e que não tinham Bacabal - MA como município de notificação.

Para a análise dos dados quantitativos foi empregada a técnica de análise estatística dos dados, os quais foram apresentados em frequência absoluta e relativa percentual, sendo utilizado o software Microsoft Excel versão 2013 para a tabulação dos dados e elaboração de gráficos e tabelas, de modo a proporcionar a organização dos mesmos.

Por se tratar de uma pesquisa eletrônica em que foram utilizados dados secundários de domínio público, não houve necessidade de aprovação desta pesquisa pelo Comitê de Ética em Pesquisa (CEP).

\section{RESULTADOS E DISCUSSÃO}

No período de 2015 a 2017, foram notificados ao SINAN 265 casos de hanseníase no município, sendo verificado o maior número no ano de 2017, que registrou 96 casos (36\%). O ano de 2016 apresentou o menor número dentre os três anos avaliados, com um total de 81 casos $(31 \%)$ notificados (Tabela 1). 
Tabela 1 - Número de casos de hanseníase notificados. Bacabal - MA, 2015 - 2017.

\begin{tabular}{ccc}
\hline Ano de Notificação & № de casos notificados & $\%$ \\
\hline 2015 & 88 & 33 \\
2016 & 81 & 31 \\
2017 & 96 & 36 \\
\hline Total & 265 & 100 \\
\hline
\end{tabular}

Fonte: SILVA PSR, et al., 2020; dados extraídos do Sistema de Informação de Agravos de Notificação - SINAN, 2020.

Considerando a taxa de detecção de casos novos de hanseníase, no ano de 2017 o munícipio apresentou uma taxa de detecção de 53,21 casos por 100 mil habitantes, o que, segundo parâmetros estabelecidos pelo Ministério da Saúde (2016), o caracteriza como hiperendêmico para a hanseníase, uma vez que a taxa calculada atingiu um valor superior a 40 casos por 100 mil habitantes. Os dados referentes aos anos de 2015 e 2016 não se encontram disponíveis no sistema.

Analisando os aspectos sociodemográficos e clínicos dos pacientes portadores de hanseníase, verificase o predomínio da doença no sexo masculino com 168 casos (63\%) durante o período avaliado, sendo identificado o maior número de casos na faixa etária de 30 a 39 anos, correspondendo a 53 casos (20\%). Quanto à classificação operacional da hanseníase, prevaleceu neste estudo o registro de pacientes multibacilares, com 237 casos (89\%), e na análise da variável "forma clínica", a forma dimorfa se sobressaiu em relação às demais, registrando 176 casos $(66,4 \%)$ no total (Tabela 2). 
Tabela 2 - Caracterização sociodemográfica e clínica de pacientes portadores de hanseníase. Bacabal - MA, 2015 - 2017.

\begin{tabular}{lcc}
\hline Variável & N & $\%$ \\
\hline Sexo & 168 & 63 \\
\hline Masculino & 97 & 37 \\
Feminino & 2 & 1 \\
\hline Idade & 7 & 3 \\
\hline 1 a 4 anos & 10 & 4 \\
5 a 9 anos & 10 & 4 \\
10 a 14 anos & 35 & 13 \\
15 a 19 anos & 53 & 20 \\
20 a 29 anos & 34 & 13 \\
30 a 39 anos & 41 & 15 \\
40 a 49 anos & 40 & 15 \\
50 a 59 anos & 24 & 9 \\
60 a 69 anos & 9 & 3 \\
70 a 79 anos & &
\end{tabular}

\section{Classificação operacional}

\begin{tabular}{lcc}
\hline Paucibacilar & 28 & 11 \\
Multibacilar & 237 & 89 \\
\hline Forma clínica & 9 & 3,4 \\
\hline Indeterminada & 19 & 7,2 \\
Tuberculoide & 176 & 66,4 \\
Dimorfa & 49 & 18,5 \\
Virchowiana & 11 & 4,1 \\
Não classificada & 1 & 0,4 \\
Ignorada & 265 & 100
\end{tabular}

Fonte: Silva PSR, et al., 2020; dados extraídos do Sistema de Informação de Agravos de Notificação - SINAN, 2020.

Observa- se que houve predomínio da doença em indivíduos do sexo masculino durante o período estudado. Resultados semelhantes foram encontrados em estudos sobre o perfil epidemiológico da hanseníase desenvolvidos por Vieira MS, et al. (2015) no município de União no estado do Piauí e por Mendonça CAS (2018) no estado do Maranhão, em que prevaleceram os casos no sexo masculino, com $57,5 \%$ e $56,79 \%$, respectivamente. 
Barbosa DRM, et al. (2014) afirmam que a maior ocorrência de hanseníase em indivíduos do sexo masculino se dá em virtude de os mesmos possuírem maior contato inter-humano nos espaços de trabalho, de modo que ficam mais expostos ao bacilo e, consequentemente, são mais vulneráveis ao acometimento pela doença.

Além disso, os homens detêm menor preocupação com questões relacionadas à sua saúde e raramente buscam os serviços de saúde, ocasionando, assim, um retardo no diagnóstico e aumentando o risco de desenvolvimento de deformidades e incapacidade físicas.

Nota-se que as faixas etárias mais afetadas pela hanseníase são aquelas que englobam a população adulta, sendo verificado o maior número de casos na faixa de 30 a 39 anos de idade (53 casos). Por outro lado, as faixas etárias correspondentes à população infantil registraram os menores valores, tendo a faixa de 1 a 4 anos o menor número de indivíduos acometidos pela doença (2 casos).

De acordo com Oliveira JCF, et al. (2014), devido ao fato de a hanseníase possuir um longo período de incubação, que geralmente varia de 2 a 7 anos, a doença é identificada mais comumente em indivíduos adultos, os quais acabam tendo sua capacidade de execução das atividades diárias prejudicada e, por estarem dentro da população considerada economicamente ativa, o acometimento pela hanseníase traz como resultado perdas econômicas e sociais.

É importante mencionar também a ocorrência da hanseníase na população idosa, definida pelo Ministério da Saúde como aqueles que possuem 60 anos ou mais, e que no presente estudo somaram 73 casos (27\%).

Por já vivenciarem o processo de envelhecimento e com a presença de outras comorbidades que agravam o seu estado de saúde, o adoecimento pela hanseníase aumenta o risco para o surgimento de complicações como incapacidades e deformidades físicas e por isso deve-se ter uma atenção especial a esse grupo, a fim de tentar reduzir o número de casos de hanseníase nos idosos e evitar o agravamento da doença naqueles que já são portadores da enfermidade.

Destaca-se a maior ocorrência de casos multibacilares de hanseníase durante o período da pesquisa. Em outros estudos também estão presentes dados que indicam uma maior parcela de pacientes multibacilares, como a pesquisa realizada por Lira TB, et al. (2019) sobre o perfil da hanseníase no estado do Piauí, por Campos MRM, et al. (2018) referente ao perfil epidemiológico da hanseníase na Paraíba e no Brasil e por Cruz KRP, et al. (2018), que analisaram o perfil da hanseníase na Paraíba. Em todos eles, foi constatada a prevalência da hanseníase na forma multibacilar como o grupo de contágio da doença.

Segundo Goiabeira YNLA, et al. (2018), indivíduos que possuem a forma multibacilar da hanseníase são os responsáveis pelo alto potencial de transmissão da doença, eliminando o bacilo no ambiente e infectando os indivíduos previamente saudáveis. Oliveira JCF, et al. (2014) acrescentam que a presença de uma grande quantidade de casos multibacilares acarreta o aumento da endemia, uma vez que mais pessoas estão sujeitas a adquirirem a hanseníase.

A partir dos resultados mostrados na tabela, verifica-se que no município prevalecem as formas clínicas multibacilares, dimorfa e virchowiana, que são consideradas as formas mais graves da doença e capazes de levar ao surgimento de deformidades e incapacidades físicas quando não tratadas, sendo responsáveis pelo alto potencial de transmissão da hanseníase, em razão da alta carga de bacilos eliminada pelos pacientes no ambiente, o que favorece o aumento do número de casos na região.

O elevado número de pacientes dimorfos e virchowianos e a baixa quantidade de casos de hanseníase indeterminada e tuberculoide sugerem que o diagnóstico está sendo feito tardiamente, tendo em vista que na maioria dos casos notificados a doença não foi detectada em seus estágios iniciais. As formas clínicas dimorfa e virchowiana representam as formas mais contagiosas da hanseníase e são capazes de ocasionar deformidades e incapacidades físicas com a sua evolução, além de implicarem a perpetuação da transmissão do bacilo de Hansen. A sua identificação sugere um diagnóstico tardio e demora no início do tratamento (OLIVEIRA JCF, et al., 2014; GROSSI MAF e LYON S, 2014). 
Em alguns casos a forma clínica não foi identificada no momento do diagnóstico, sendo preenchida no campo como "não classificada". Tal situação ocorre geralmente quando o profissional médico tem dúvidas quanto à definição da forma clínica presente no paciente, já que, por vezes, as mesmas podem apresentar características semelhantes, inviabilizando o estabelecimento do diagnóstico.

Também pode sugerir o despreparo do profissional em identificar as formas clínicas da hanseníase, o que, de acordo com Velôso DS, et al. (2018), pode ocasionar sérios problemas ao paciente, uma vez que um diagnóstico errado pode fazer com que ele receba o tratamento inapropriado para o seu caso clínico e, consequentemente, agravar o seu estado de saúde, além de elevar o índice de transmissão quando realizado tardiamente.

Observa-se que há uma discordância no somatório das formas clínicas em relação à classificação operacional no que diz respeito aos casos multibacilares, uma vez que estes representam 237 casos, enquanto a soma das formas dimorfa e virchowiana resulta em 225 casos, o que se deve ao fato de que os dados restantes (12 casos) foram adicionados aos itens "não classificado" e "ignorado" da variável "forma clínica", completando, assim, os 237 casos considerados multibacilares.

Considerando o modo de entrada dos pacientes no sistema, os dados mostram que 182 casos (68,7\%), compreendendo a maioria, foram registrados como caso novo, definido pelo Ministério da Saúde (2017a) como aquele que nunca recebeu qualquer tratamento específico para a doença, seguido de 30 casos $(11,3 \%)$ de transferência do mesmo município, 6 casos $(2,3 \%)$ de recidiva, 2 casos $(0,7 \%)$ de transferência de outro estado e 1 caso $(0,4 \%)$ de transferência de outro município (mesma UF). Outros ingressos representaram 44 casos (16,6\%) notificados (Gráfico 1).

Gráfico 1 - Casos de hanseníase de acordo com o modo de entrada. Bacabal - MA, 2015 - 2017

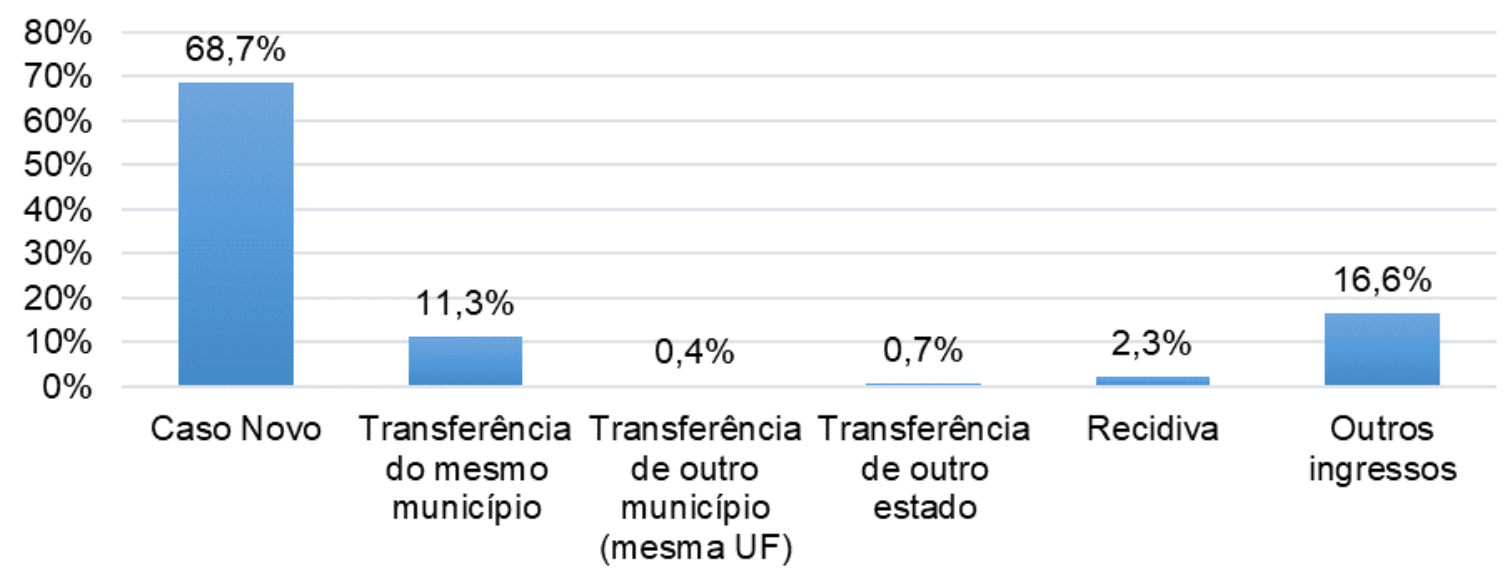

Fonte: Silva PSR, et al., 2020; dados extraídos do Sistema de Informação de Agravos de Notificação - SINAN, 2020.

O elevado número de casos novos detectados permite inferir um aumento da incidência da hanseníase na região durante o período avaliado, evidenciando falhas nas ações de controle da doença por parte dos serviços de saúde e órgãos que atuam na vigilância da hanseníase. Destaca-se, por outro lado, uma baixa ocorrência de casos de recidiva da doença, o que significa que a maior parte dos pacientes conseguiu responder bem à terapia instituída, eliminando o bacilo totalmente do organismo, tendo em vista que, de acordo com Bona SH, et al. (2015), dentre os principais fatores relacionados à ocorrência de recidiva estão a persistência bacilar, a resistência medicamentosa e erros na classificação operacional.

No que diz respeito ao modo de detecção da hanseníase, pode-se observar que a maioria dos casos foram identificados por demanda espontânea, que registrou 95 casos $(36 \%)$, seguido de detecção por encaminhamento, com 82 casos (31\%), 3 casos (1\%) identificados a partir da realização de exame de contatos e 2 casos $(0,7 \%)$ detectados em exame de coletividade. Em 83 casos (31,3\%), a variável "modo de detecção" foi registrada como "ignorado" (Gráfico 2). 
Gráfico 2 - Casos de hanseníase conforme o modo de detecção. Bacabal - MA, 2015 - 2017.

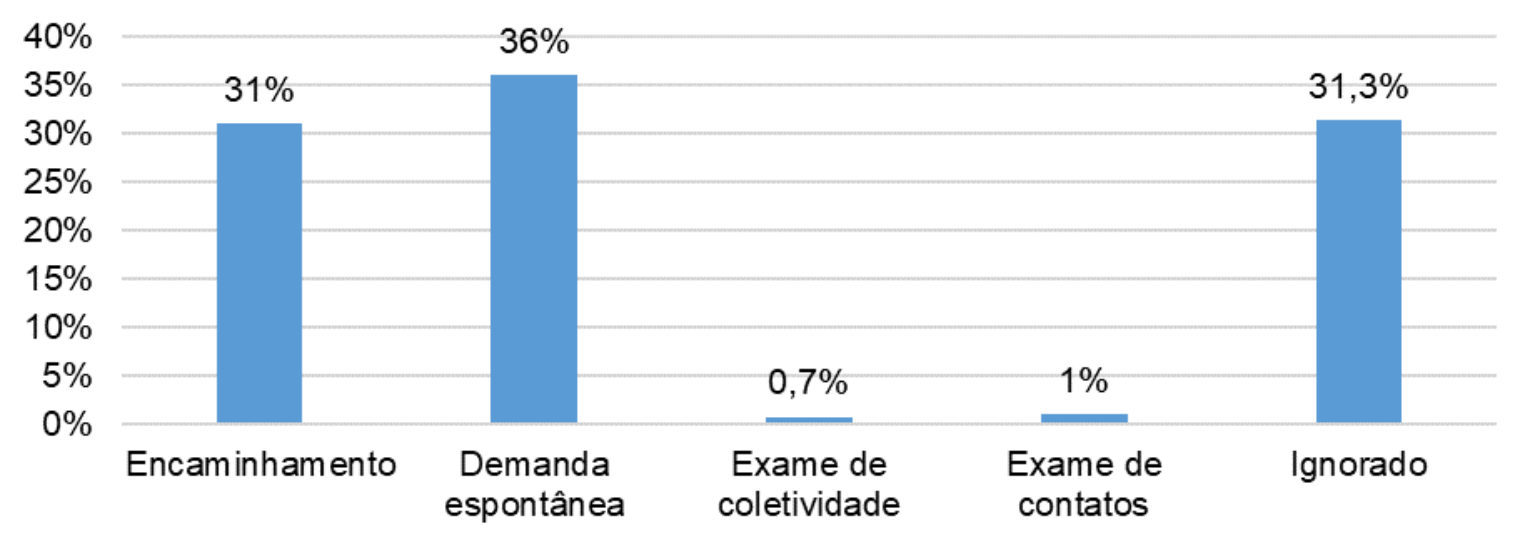

Fonte: Silva PSR, et al., 2020; dados extraídos do Sistema de Informação de Agravos de Notificação - SINAN, 2020.

Observa-se que apesar da detecção por meio de demanda espontânea ter sido elevada, a detecção através do exame de contatos e de coletividade, ao contrário, foi baixa, o que permite inferir que a busca ativa foi pouco implementada pelos profissionais nos serviços de saúde do município durante o período avaliado. O Ministério da Saúde (2016) destaca que a vigilância de contatos representa a principal estratégia de detecção ativa para a descoberta de casos, possibilitando o diagnóstico precoce e a queda da cadeia de transmissão da doença, com consequente redução das deficiências provocadas pela hanseníase. Diante disso, faz-se necessário que as ações de busca ativa sejam mais priorizadas pelos profissionais de saúde, por meio do conhecimento de casos suspeitos, detecção precoce de portadores de hanseníase e prevenção da doença em indivíduos saudáveis, para que, dessa forma, seja executado o controle efetivo do agravo e se alcance a eliminação da hanseníase como problema de saúde pública.

Quanto à avaliação do grau de incapacidade física (GIF), no momento do diagnóstico a maior parte dos pacientes apresentou grau 0 de incapacidade, compreendendo 116 casos (44\%), seguido por 93 casos (35\%) com presença de grau 1 de incapacidade e 27 casos (10\%) com grau 2 de incapacidade física, representando, assim, a minoria dos casos com o GIF avaliado. 24 casos (9\%), não tiveram o grau de incapacidade física avaliado durante o diagnóstico e em 5 casos (2\%), o item em questão foi deixado em branco (Gráfico 3).

Gráfico 3 - Casos de hanseníase segundo avaliação do grau de incapacidade física no diagnóstico. Bacabal - MA, 2015 - 2017.

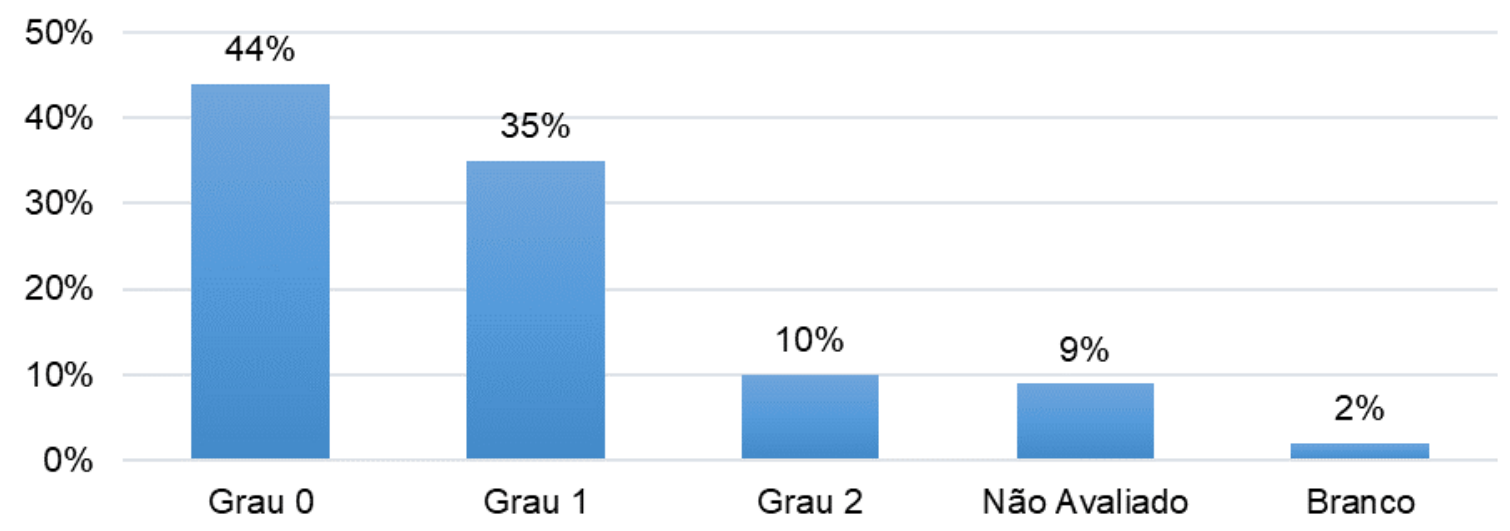

Fonte: Silva PSR, et al., 2020; dados extraídos do Sistema de Informação de Agravos de Notificação - SINAN, 2020. 
Tais resultados corroboram com uma pesquisa realizada por Santos GRB, et al. (2018), que avaliou o perfil da hanseníase em São luís - MA, onde $68,7 \%$ dos casos apresentaram grau 0 de incapacidade física no diagnóstico. Outro trabalho foi realizado por Sarmento APA, et al. (2015) em Montes Claros, no estado de Minas Gerais, no qual os dados demonstraram que a maioria dos pacientes apresentou, no momento do diagnóstico, grau 0 de incapacidade. Observa-se que, embora tenha predominado o número de pacientes com GIF 0, a quantidade de pacientes que apresentaram algum grau de incapacidade física no momento do diagnóstico foi levemente superior (120 casos), evidenciando que houve demora na realização do diagnóstico, visto que, quanto mais cedo se detecta um caso de hanseníase, menores são as chances de o paciente vir a desenvolver incapacidades físicas.

Alguns casos não tiveram o GIF avaliado no momento do diagnóstico, o que sugere falha dos profissionais de saúde na assistência prestada ao paciente com hanseníase ou mesmo despreparo dos mesmos em executar a avaliação. Carvalho NV e Araújo TME (2015) apontam que é essencial realizar a avaliação do grau de incapacidade física no momento do diagnóstico, a fim de identificar a presença de deformidades e incapacidades físicas nos indivíduos acometidos por hanseníase, buscando prevenir precocemente o surgimento de danos em pacientes que ainda não possuem tais incapacidades e tratar aqueles em que elas já estão presentes. Na análise da variável "tipo de saída", 143 casos (54\%) tiveram sua saída registrada por cura, constituindo a maioria, seguido de 43 casos $(16,2 \%)$ de transferência para o mesmo município, 9 casos $(3,4 \%)$ de transferência para outro município, 6 casos $(2,3 \%)$ de óbito, 4 casos $(1,5 \%)$ de abandono do tratamento, 2 casos $(0,7 \%)$ de erro diagnóstico e 1 caso $(0,4 \%)$ de transferência para outro estado. Em 57 casos (21,5\%) o item "tipo de saída" foi registrado como "ignorado" (Gráfico 4).

Gráfico 4 - Casos de hanseníase conforme o tipo de saída. Bacabal - MA, 2015 - 2017.

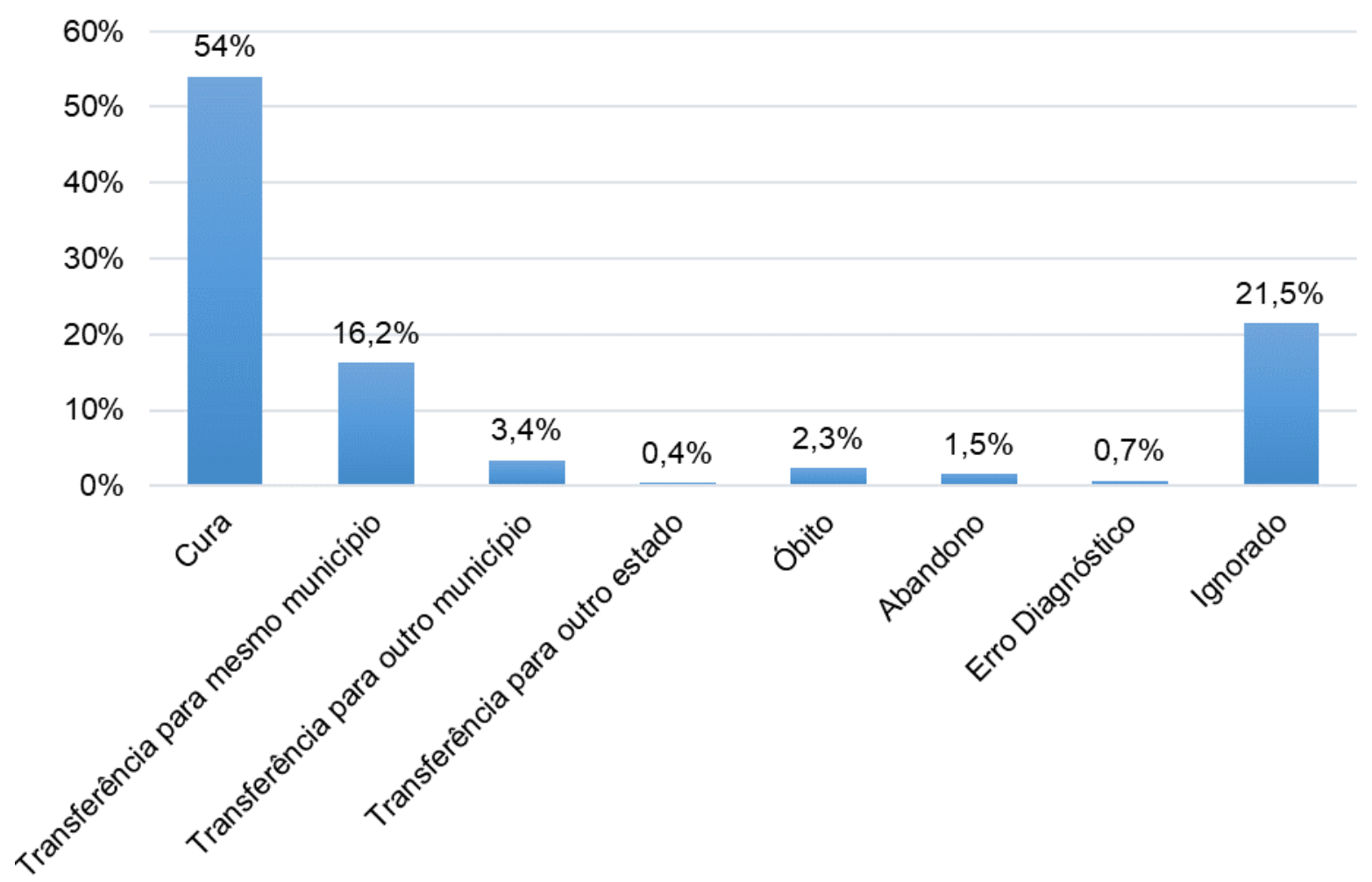

Fonte: Silva PSR, et al., 2020; dados extraídos do Sistema de Informação de Agravos de Notificação - SINAN, 2020.

Os resultados relacionados à variável "tipo de saída" mostraram-se satisfatórios, uma vez que a maior parte dos pacientes realizou o tratamento com a poliquimioterapia até o final e com isso receberam a alta por cura, indicando que os mesmos tiveram uma boa adesão ao tratamento. Além disso, o número de casos em abandono de tratamento foi pequeno, o que reforça o compromisso dos indivíduos acometidos por 
hanseníase em alcançar a cura da doença por meio da aceitação e do correto seguimento da terapêutica instituída. Importante frisar os casos de óbito de pacientes, possivelmente em decorrência de complicações da hanseníase, sendo registrados poucos casos durante o período da pesquisa, o que é considerado normal, tendo em vista que a mortalidade pela hanseníase geralmente é baixa.

O elevado número de ignorados é um indicativo de falha no registro dos dados referentes ao tipo de saída do sistema de informação, reforçando novamente a necessidade de maior empenho por parte dos profissionais de saúde em realizar o correto preenchimento das informações requeridas nas fichas. Diante do perfil traçado, sugere-se a necessidade de se intensificarem as ações de controle da hanseníase nos serviços de saúde, por meio da adoção de medidas como a detecção precoce e tratamento oportuno dos casos diagnosticados, maior enfoque nas ações de busca ativa, bem como promoção de capacitações para os profissionais de saúde, sobretudo àqueles responsáveis pelo atendimento direto ao indivíduo acometido pela hanseníase, a fim de que estejam preparados para executarem adequadamente a avaliação dermatoneurológica e a avaliação do grau de incapacidade física dos pacientes, além de realizarem o correto preenchimento das fichas de hanseníase (ANDRADE YNL, 2016).

Atenção deve ser dada também às ações de educação em saúde para a população, assegurando o repasse de informações acerca dos sinais e sintomas da doença, a importância do diagnóstico e tratamento oportunos, o estímulo à regularidade do tratamento, além de eliminar ideias errôneas associadas à hanseníase (BRASIL, 2016).

Colocando em prática tais intervenções, será possível obter bons resultados no controle da doença no município, possibilitando a redução do número de casos e interrupção da sua cadeia de transmissão, auxiliando na eliminação da hanseníase como problema de saúde pública.

\section{CONSIDERAÇÕES FINAIS}

A hanseníase continua sendo um problema de saúde pública na região, uma vez que o município ainda é considerado hiperendêmico para a doença. Foram atingidos predominantemente indivíduos do sexo masculino, a faixa etária de 30 a 39 anos, com maior número de pacientes multibacilares, sendo a forma clínica dimorfa a mais frequente. Quanto ao modo de entrada, houve predomínio de indivíduos registrados como caso novo, o modo de detecção se deu principalmente por demanda espontânea, a maioria dos casos avaliados apresentou grau 0 de incapacidade ao diagnóstico e a maior parte teve a saída registrada como cura. O presente estudo forneceu um panorama acerca da situação da hanseníase no município, o que permite contribuir para a sua monitorização e para a elaboração e implementação de medidas de redução do agravo e enfrentamento da doença.

\section{REFERÊNCIAS}

1. ANDRADE YNL. Indicadores de qualidade das ações e serviços de saúde do Programa de Controle da Hanseníase em capital hiperendêmica no Brasil. Dissertação (Mestrado em Enfermagem) - Universidade Federal do Maranhão, São Luís, 2016; 84 p.

2. BARBOSA DRM, et al. Características epidemiológicas e espaciais da hanseníase no Estado do Maranhão, Brasil, 2001-2012. Revista Medicina USP, 2014; 47(4): 347-356.

3. BONA SH, et al. Recidivas de hanseníase em Centros de Referência de Teresina, Piauí, 2001-2008. Epidemiologia e Serviços de Saúde, 2015; 24(4): 731-738.

4. BRASIL. Ministério da Saúde. Secretaria de Vigilância em Saúde. Boletim epidemiológico: Hanseníase. Brasília: Ministério da Saúde. 2018; 49(4): 12.

5. BRASIL. Ministério da Saúde. Secretaria de Vigilância em Saúde. Departamento de Vigilância das Doenças Transmissíveis. Diretrizes para vigilância, atenção e eliminação da Hanseníase como problema de saúde pública: manual técnico-operacional. Brasília: Ministério da Saúde, 2016, 60 p.

6. BRASIL. Ministério da Saúde. Secretaria de Vigilância em Saúde. Coordenação- Geral de Desenvolvimento da Epidemiologia em Serviços. Guia de Vigilância em Saúde: volume único. 2. ed. Brasília: Ministério da Saúde, 2017a. 706 p. 
7. BRASIL. Ministério da Saúde. Secretaria de Vigilância em Saúde. Departamento de Vigilância das Doenças Transmissíveis. Guia prático sobre a hanseníase. Brasília: Ministério da Saúde, 2017b. 70 p.

8. CAMPOS MRM, et al. Perfil Clínico-Epidemiológico dos Pacientes Diagnosticados com Hanseníase na Paraíba e no Brasil, 2008-2012. Revista Brasileira de Ciências da Saúde, 2018; 22(1): 79-86.

9. CARVALHO NV, ARAÚJO TME. Ações realizadas por profissionais de Saúde da Família no controle da hanseníase em um município hiperendêmico. Journal of Health and Biological Sciences, 2015; 3(3): 144-150.

10. COÊLHO LS, et al. Vivência do enfermeiro da atenção básica nas ações de controle da hanseníase. Revista de Enfermagem UFPE online, 2015; 9(10): 1411-1417.

11. CRUZ KRP, et al. Avaliação epidemiológica dos casos de hanseníase no estado da Paraíba. In: III Congresso Brasileiro de Ciências da Saúde, Campina Grande, 2018.

12. DATASUS. Hanseníase-Maranhão. http://tabnet.datasus.gov.br/cgi/dhdat.exe?hanseniase/hantfma17.def. Acesso em: 26 jul. 2019.

13. GOIABEIRA YNLA, et al. Perfil epidemiológico e clínico da hanseníase em capital hiperendêmica. Revista de Enfermagem UFPE online, 2018; 12(6): 1507-1513.

14. GROSSI MAF, LYON S. Diagnóstico e tratamento da hanseníase. In: ALVES ED, et al. (Org.). Hanseníase: avanços e desafios. Brasília: NESPROM, 2014; 494 p.

15. LIRA TB, et al. Hanseníase no Piauí: uma investigação epidemiológica. Revista Eletrônica Acervo Saúde, 2019; (24): e499.

16. MACIEL LR, FERREIRA IN. A presença da hanseníase no Brasil - alguns aspectos relevantes nessa trajetória. In: ALVES ED, et al. (Org.). Hanseníase: avanços e desafios. Brasília: NESPROM, 2014; 494 p.

17. MENDONÇA CAS. Perfil clínico- epidemiológico dos pacientes diagnosticados com hanseníase no estado do Maranhão de 2006 a 2015. Monografia (Graduação em Enfermagem) - Universidade Federal do Maranhão, São Luís, 2018; $55 \mathrm{p}$.

18. NASCIMENTO GRC, et al. Ações do enfermeiro no controle da hanseníase. Revista Eletrônica de Enfermagem, $2011 ; 13(4): 743-50$.

19. OLIVEIRA JCF, et al. Análise do perfil epidemiológico da hanseníase em Maricá, Rio de Janeiro: uma contribuição da enfermagem. Revista de Enfermagem UERJ, 2014; 22(6): 815-821.

20. SANTOS GRB, et al. Prevalência de hanseníase em São Luís - Maranhão entre os anos de 2013 a 2015. Journal of Nursing and Health, 2018; 8(2): 01-13.

21. SARMENTO APA, et al. Perfil epidemiológico da hanseníase no período de 2009 a 2013 no município de Montes Claros (MG). Revista Sociedade Brasileira de Clínica Médica, 2015; 13(3): 180-184.

22. VELÔSO DS, et al. Perfil Clínico Epidemiológico da Hanseníase: Uma Revisão Integrativa. Revista Eletrônica Acervo Saúde. 2018; 10(1): 1429-1437.

23. VIEIRA MS, et al. Perfil epidemiológico da hanseníase no município de União-PI no período de 2010 a 2013. Revista Interdisciplinar. 2015; 8(4): 120-126. 